\title{
Immunogenetic studies of maternal-fetal relationships: a review: why newborn rhesus monkeys don't get hemolytic disease
}

\author{
R. S. Treichel \\ Department of Genetics, Southwest Foundation for Biomedical Research, San Antonio, TX 78284, USA
}

\begin{abstract}
The discovery of the Rh blood group factor in humans was made using the red blood cells of rhesus monkeys. Because of its importance to human medicine and immunogenetics, this finding contributed greatly to the appreciation of the importance of nonhuman primates in research. It is now widely recognized that blood group incompatibility between mother and fetus can lead to differential fertility, fetal death, and hemolytic disease of the newborn (HDN).

The blood group systems of several nonhuman primate species have been studied in detail and found to be analogous, although not identical, to those of humans. It is therefore surprising that HDN has been reported in only four nonhuman primate species - marmosets, sacred baboons, chimpanzees, and orangutans. Maternal-fetal blood group incompatibility and its consequences have been extensively studied in rhesus monkeys, and these macaques may well be representative of many nonhuman primates. Rhesus monkeys exhibit all five of the conditions that lead to HDN in humans: (1) blood group incompatible matings; (2) transplacental hemorrhage; (3) maternal immunization to blood group alloantigens on fetal erythrocytes; (4) transplacental transfer of maternal antibodies; and (5) coating of the newborn's erythrocytes. Yet, newborns show no clinical or hematological evidence of HDN.

We have shown that the rhesus alloantibodies engendered by transplacental immunization do not mediate immune elimination of the newborn's erythrocytes. Evaluation of the maternal antibodies demonstrated that they have low titers and low avidities and perhaps belong to IgG subclasses that do not bind effectively to receptors on phagocytic cells of the rhesus reticuloendothelial system. The newborn's genotype may also affect the expression of allogeneic blood group antigens and thereby help protect the newborn's cells from destruction. These factors together undoubtedly play a major role in the survival of the antibody-coated newborn's RBC and are thus able to account for the absence of HDN in this species.
\end{abstract}

\section{Introduction}

The brilliant analysis of $\mathrm{Rh}$ hemolytic disease of the newborn (HDN) in humans clearly demonstrated the immunogenetic significance of the maternalfetal relationship. We now have abundant evidence that incompatibility between mother and fetus for the $\mathrm{Rh}, \mathrm{ABO}$ and other blood group systems can lead to differential fertility, fetal death and hemolytic disease of the newborn (Hildemann et al., 1981). Clearly, natural selection acts through maternal-fetal incompatibility to influence the frequencies of blood group alleles and phenotypes in populations.

HDN, also known as erythroblastosis fetalis, occurs when a woman becomes immunized to antigens carried by fetal red blood cells (RBC). These antibodies cross the placenta, coat the fetal RBC and mediate their destruction. If the fetus cannot generate new $\mathrm{RBC}$ rapidly enough to replace those being destroyed, anemia results. In severe form, the fetus will die in utero and may be hydropic or 
macerated. More commonly, manifestations of the disease are expressed during the neonatal period as decreased hemoglobin and hematocrit values, elevated bilirubin levels, and increased reticulocyte counts (hence the term 'erythroblastosis fetalis'). The major complication of the disease is jaundice, which increases in severity after the baby is born.

HDN occurs not only in humans but also in several mammalian species (Roberts, 1957) and in chickens (Briles, 1948). However, only in humans, certain nonhuman primates, horses and mules does the disease occur spontaneously with immunization resulting solely from pregnancy. In other species, maternal antibodies are engendered by incompatible transfusions, overt immunizations, or the use of vaccines containing blood or blood products. Various species also differ markedly with respect to the time and route of transfer of the maternal antibodies. In rabbits, as in humans, maternal antibodies are transferred to the fetus prior to birth, but via the yolk sac rather than the placenta. In horses, dogs, pigs, and cattle, maternal antibodies cannot cross the placenta but rather are ingested in colostrum by the newborn. Because HDN appears only after nursing in these species, it is commonly termed 'neonatal isoerythrolysis' (Stormont, 1975).

\section{Hemolytic disease of the newborn in nonhuman primates}

During the past twenty years knowledge of the blood groups of nonhuman primates has grown immensely, thanks to the availability of pedigreed breeding colonies and to the development of reagents capable of detecting allogeneic differences. The discovery of blood groups in nonhuman primates (for review see Stone, 1975; Socha \& Ruffie, 1983) raised the question of whether maternal-fetal incompatibility results in HDN in these animals, as might be expected because the placentas of primates are structurally similar and are selectively permeable to gamma globulin (Rodewald, 1980). However, contrary to expectation, HDN has been documented in only a few species of nonhuman primates. Several studies that highlight the immunogenetic aspects of HDN in these primates will be discussed briefly.

HDN occurs spontaneously in the marmoset
Tamarinus nigricollis (also known as Saquinus nigricollis), and may be a major cause of the abortions, stillbirths and neonatal deaths frequently observed in this species. Histopathological and serological evidence of the disease were reported for twins delivered by two different mothers (Gengozian et al., 1966). The sera of each mother contained antibodies reactive with her mate's cells. Twinning is very common in marmosets and the resulting blood chimerism of the offspring can make blood group determinations difficult. Nevertheless, maternal antibodies could be demonstrated on the $\mathrm{RBC}$ of the one set of twins tested. In S. fuscicollis, a mother who had been previously immunized with allogeneic RBC miscarried twin fetuses. Antibodies present in her serum reacted strongiy with the twins' RBC (Gengozian, 1972). The author ascribed the deaths to HDN; unfortunately, he did not report histopathological evidence for the diagnosis.

Russian researchers (Verbickij, 1972) developed an experimental model of HDN in sacred baboons (Papio hamadryas hamadryas). In a study conducted at the Sukhumi Primate Center, USSR, a total of 52 females were immunized before and during their pregnancies with RBC of their mates. Five pregnancies resulted in miscarriages and 21 in stillbirths. Of the 26 liveborn infants, 11 had jaundice and eight were anemic. Hematological and histopathological changes found in the affected baboons were very similar to those in affected human infants, and in addition, their cells gave positive direct antiglobulin (DAT) reactions. Notably, seven newborns showed no clinical signs of the disease; however, it is unclear whether the cells of these unaffected infants were coated with maternal antibody. The authors did state that the severity of the disease process did not correlate absolutely with the maternal antibody titer.

Baboons in the Sukhumi breeding colony also exhibited spontaneous HDN resulting from transplacental rather than overt immunization of the mother (Verbitsky et al., 1969). Clinical, hematological, and histopathological features of the spontaneous disease were indistinguishable from the experimental model. In both situations blood group alloantibodies could be detected in maternal sera. The authors failed to address the question of whether an immunodominant baboon blood group factor or system accounted for the spontaneous 
disease, as is the case with the $\mathrm{D}$ antigen in humans which accounts for most clinically recognized HDN. Nevertheless, based on their studies of 1127 pregnancies, they concluded that $16 \%$ had pathological outcomes.

It is of interest that the results for sacred baboons conflict with those for olive baboons ( $P$. hamadryas cynocephalus); HDN has not been observed in the large breeding colony of olive baboons maintained at the Southwest Foundation for Biomedical Research. Moreover, HDN could not be induced by hyperimmunizing four olive baboons and sacred baboons during pregnancy with red cells of their mates (Socha \& Ruffie, 1983). Although the cells of two infants gave a moderate direct antiglobulin reaction, no clinical symptoms of HDN were observed.

Three reported pregnancies indicated that maternal-fetal blood group incompatibility can cause erythroblastosis fetalis in chimpanzees (Wiener et al., 1977). One liveborn infant exhibited severe jaundice, one died shortly after birth with histological evidence of HDN, the third pregnancy resulted in the birth of a macerated fetus. Antibodies present in the serum of each mother reacted with the infant's or mate's cells and notably increased in titer during pregnancy. Serological analysis indicated that in two cases the $\mathrm{R}^{\mathrm{c}}$ alloantigen, a chimpanzee blood group factor which is related to the human $\mathrm{Rh}$ antigens, was responsible for the observed maternal-fetal incompatibility; whereas the $\mathrm{V}^{c}$ specificity was most likely involved in the third case. Significantly, all three mothers had been previously immunized against the incompatible blood group factor. Apparently, HDN does not occur spontaneously in chimpanzees, although only the relatively small number of chimpanzees bred in captivity has been studied to date.

Symptoms of severe HDN including marked jaundice and anemia were observed in a fullterm orangutan infant, the fourth offspring of a single breeding couple (Socha \& Van Foreest, 1981). It is probable that repeated incompatible pregnancies stimulated the high titer of antibodies detected in the maternal serum. The newborn's RBC were heavily coated, and free antibodies were detected in its serum. Extensive serological analysis of the mother's postpartum serum and of an eluate from the newborn's cells indicated transplacental immunization may have been induced by an orangutan factor serologically related to, although not identical with, the human D antigen.

HDN has not been reported in rhesus monkeys (Macaca mulatta), even though the human Rh factor was originally detected using a rabbit antiserum produced against RBC from rhesus monkeys (Landsteiner \& Wiener, 1940). In fact, experimental induction of HDN was attempted in four rhesus macaques without success (Sullivan et al., 1976). Although the hyperimmunized pregnant females produced potent antibodies which crossed the placenta and coated the newborn's RBC, no clinical or hematological evidence of HDN was observed. Similar experiments by other investigators (Wiener et al., 1975; Socha \& Moor-Jankowski, 1979) also failed to induce clinical evidence of HDN in rhesus or crab-eating macaques ( $M$. fascicularis). These results were unexpected because it had been believed that maternal-fetal blood group incompatibility might account for some of the 12-25\% spontaneous abortions and stillbirths observed in breeding colonies of rhesus monkeys (Stone, 1975). These results also demonstrated the importance of histopathological and serological documentation before ascribing fetal and neonatal deaths to HDN.

The inability to induce HDN in rhesus monkeys suggested that some mechanism was 'protecting' the coated cells from destruction. Several species possess mechanisms to protect against HDN; these operate to prevent maternal immunization to fetal $\mathrm{RBC}$ or to prevent maternal antibodies from effectively opsonizing fetal cells (Voak, 1969; Clarke, 1978; Stormont, 1975). The following is a summary of our progress in attempting to elucidate the "protective mechanism' of rhesus monkeys. This paper is not intended to be a comprehensive review, but rather a highlight of those discoveries that are particularly relevant to the question: Why don't rhesus monkeys develop HDN?

\section{The absence of HDN in rhesus monkeys}

The absence of HDN in rhesus monkeys is not due to an absence of polymorphic blood group systems. Rhesus monkeys possess allogeneic blood group systems which are serologically and genetically analogous, though not identical, to those of humans. The Immunogenetics Laboratory at the University of Wisconsin has developed 32 alloim- 
mune monospecific typing sera that detect 21 antigens belonging to 13 genetically-independent systems (loci) as well as nine additional factors which have not yet been assigned to genetic systems. Eleven of the 13 defined systems contain a null allele which does not code for a serologically detectable product (factor) (Stone et al., 1981).

Surprisingly, the antigen on rhesus RBC that evoked the original $\mathrm{Rh}$ typing reagent is not identical to the human Rh antigen (Levine et al., 1961). In fact, none of the typing reagents for the human $\mathrm{Rh}$ system react with rhesus $\mathrm{RBC}$ (Wiener et al., 1953). The rhesus antigen, now termed ' $L W$ ' in honor of Landsteiner and Weiner, is also present on most human RBC and there exists a large body of evidence to suggest that the $\mathrm{LW}$ and $\mathrm{Rh}$ antigens associate to form a highly complex antigenic structure on human RBC (for references see Issitt \& Issitt, 1977). The genes for LW and Rh segregat : $:$ dependently. It should be noted, however, that there is some disagreement with this interpretation. Wiener and associates believe that the antiserum originally produced by injecting rhesus RBC into guinea pigs contained anti-Rho, anti-LW and several other specificities and that rhesus RBC express an $\mathrm{Rh}$-like determinant which is masked and thus inaccessible to human anti-Rh antibodies (Wiener, et al., 1971).

\section{Transplacental immunization in rhesus monkeys}

Maternal-fetal blood group incompatibility in rhesus monkeys frequently results in transplacental alloimmunization. In fact, all the conditions that lead to red cell destruction and HDN in humans namely, incompatible matings, maternal immunization, and coating of fetal RBC - also occur in rhesus monkeys.

Over a two year period 242 successful pregnancies in the rhesus colony at the Wisconsin Regional Primate Research Center were investigated (Stong et al., 1984). Mothers and offspring were blood typed, and the results indicated that greater than $90 \%$ of the pregnancies were incompatible for one or more of the blood group factors that we can detect. To determine if this incompatibility resulted in maternal immunization, serum was collected from each mother within 14 days postpartum. Seventynine (nearly $33 \%$ ) of these postpartum sera contained agglutinins reactive with her infant's or mate's RBC. We detected fetal RBC in the circulations of 10 pregnant females by using a modification of the Kleihauer-Betke technique, which distinguishes adult and fetal RBC by taking advantage of the differential solubilities of adult and fetal hemoglobin in weak acid. Because rhesus monkeys lack soluble allogeneic blood group antigens in their serum and saliva, it is highly probable that fetal RBC that entered the maternal circulation elicited the antibodies detected in the postpartum sera.

To determine if maternal antibodies crossed the placenta and entered the fetal circulation, $R B C$ from 99 newborns were tested using the direct antiglobulin test. In 11 cases $(11 \%)$, the cells were clearly positive, indicating that maternal antibodies coated the newborn's cells. In the one case tested, low-titered maternal antibodies were detected in the newborn's serum. Yet, surprisingly, none of the newborns showed any signs or symptoms of HDN.

Our study revealed four areas in which the consequences of maternal-fetal blood group incompatibility differ between rhesus monkeys and humans:

Absence of an immunodominant factor. In humans most instances of transplacental sensitization and clinical HDN result from incompatibility for the $\mathrm{D}$ antigen of the Rh system (Giblett, 1964). In rare instances other blood group antigens of $\mathrm{Rh}$ and other systems elicit transplacental antibodies and induce HDN. In contrast, in rhesus monkeys several different blood group factors were found to elicit antibodies during pregnancy. To determine the specificity of the maternal antibodies, postpartum sera reactive with newborn's RBC were tested against a panel of rhesus RBC of known blood type. The results indicated that 9 blood group factors belonging to 6 independent systems stimulated the maternal antibodies. However, certain factors were more immunogenic than others. For example, in 7 of 29 pregnancies $(24 \%)$ in which infants were incompatible for the $\mathrm{K}$ blood group factor, the mothers produced anti-K, whereas in only $7 \%$ of $G_{1}$ - incompatible pregnancies was ant $i-G_{1}$ produced.

No parity effect. Contrary to expectation, we found that parity did not play a role in the transplacental sensitization of rhesus monkeys. The percentage of primiparous females who became sensitized $(32.4 \%)$ equaled the percentage of multiparous females who became immunized $(32.3 \%)$. In addition, we found that antibody titers 
in first incompatible pregnancies did not differ from those in subsequent incompatible pregnancies. In contrast, at least two incompatible pregnancies are usually required in humans to stimulate detectable antibodies, and once sensitization occurs, subsequent exposures to antigen induce an anamnestic rise in antibody titer (Fraser \& Tovey, 1976).

Transplacental hemorrhages. Since Levine and coworkers (1941) described the etiology of HDN, small numbers of fetal cells have been detected in the blood of pregnant women. In humans, the incidence of transplacental hemorrhage allowing fetal cells into the maternal circulation increases from $10 \%$ in early pregnancy to $20 \%$ near parturition, to approximately $50 \%$ after normal delivery (reviewed by Fraser \& Tovey, 1976). In contrast, fetal RBC were detected before and after parturition in all rhesus pregnancies. In several cases fetal cells were detected as early as the second month, and in all cases the extent and incidence of transplacental hemorrhages increased as pregnancy progressed. Our results demonstrated that transplacental hemorrhages occur earlier and with higher frequency in rhesus monkeys than in humans.

Frequency of sensitization and antibody transfer. Before the introduction of Rh prophylaxis, the incidence of maternal immunization to the $D$ factor was $0.1 \%$ for the first incompatible pregnancy and $11 \%$ for subsequent pregnancies. The $33 \%$ incidence of maternal immunization in rhesus is much higher than this. Also, whereas $11 \%$ of rhesus newborns in our study exhibited positive DAT reactions, historically only $0.5 \%$ of human newborns exhibited positive DAT reactions due to $\mathrm{Rh}$ incompatibility (Fraser \& Tovey, 1976). It is rare in humans for an immunized mother to deliver a DATnegative newborn infant. In contrast, $72 \%$ of immunized rhesus mothers delivered DAT-negative newborns, suggesting that the incidence of antibody transfer from mother to fetus is lower in rhesus than in humans. Finally, none of our rhesus newborns exhibited symptoms of HDN; in humans, $60 \%$ of infants showing positive DAT reactions due to Rh-incompatibility are affected severely enough to require exchange transfusions. Interestingly, although $5 \%$ of $\mathrm{ABO}$-incompatible human newborns exhibit DAT-positive RBC, only about 1 in 3000 has severe anemia requiring treatment (Mollison, 1983). Thus, it appears that the effects of maternal-fetal incompatibility in rhesus monkeys more closely resemble those in humans due to $\mathrm{ABO}$-incompatibility rather than $\mathrm{Rh}$ incompatibility.

\section{Cr-erythrocyte survival experiments}

The absence of HDN in rhesus despite the frequent occurrence of maternal sensitization and coating of newborns' RBC suggested that rhesus maternal antibodies were not effective in mediating erythrocyte destruction. To test this hypothesis, we studied the survival of uncoated and antibodycoated $\mathrm{RBC}$ in rhesus monkeys using $\mathrm{Na}_{2}{ }^{51} \mathrm{CrO}$ as a radioactive tracer. The details of this technique have been described previously (Stong et al., 1982). Briefly, ${ }^{5 i} \mathrm{Cr}$-labeled cells were injected as a $20 \%$ suspension via a catheter into the saphenous veins of anesthetized (Ketamine) recipients. Generally, the equivalent of about $2 \%$ of the recipient's blood volume was injected. (For example, about $18 \mathrm{ml}$ would be injected into a $10 \mathrm{~kg}$ recipient.) Certain experiments required rhesus recipients that were nonimmune. Although rhesus do not possess naturally-occuring blood group alloantibodies, prior to these experiments the sera of potential recipients were cross-matched with the donor $\mathrm{RBC}$ using our modified (Stong et al., 1982) microtiter agglutination technique (Sullivan et al., 1977) to ensure that the recipient had not been previously immunized to the donor's RBC. In the modified assay, Ficoll $(2.5 \%)$ is added to the antibody-RBC mixture so that $\mathrm{RBC}$ are agglutinated by incomplete as well as complete antibodies. At predetermined time intervals following the injection of labeled cells, blood samples were collected through the catheter. These samples were counted, both as whole blood and as separated plasma and cellular fractions. Comparison of the number of radioactive counts in the samples to the number of counts injected yielded an estimate of the percentage of injected cells still circulating at the time the sample was obtained. The finding of radioactive counts in plasma indicated that the injected cells had been lysed intravascularly.

\section{Effect of maternal antibodies on erythrocyte sur- vival}

In the first series of experiments, we compared the survival of DAT-positive RBC from two new- 


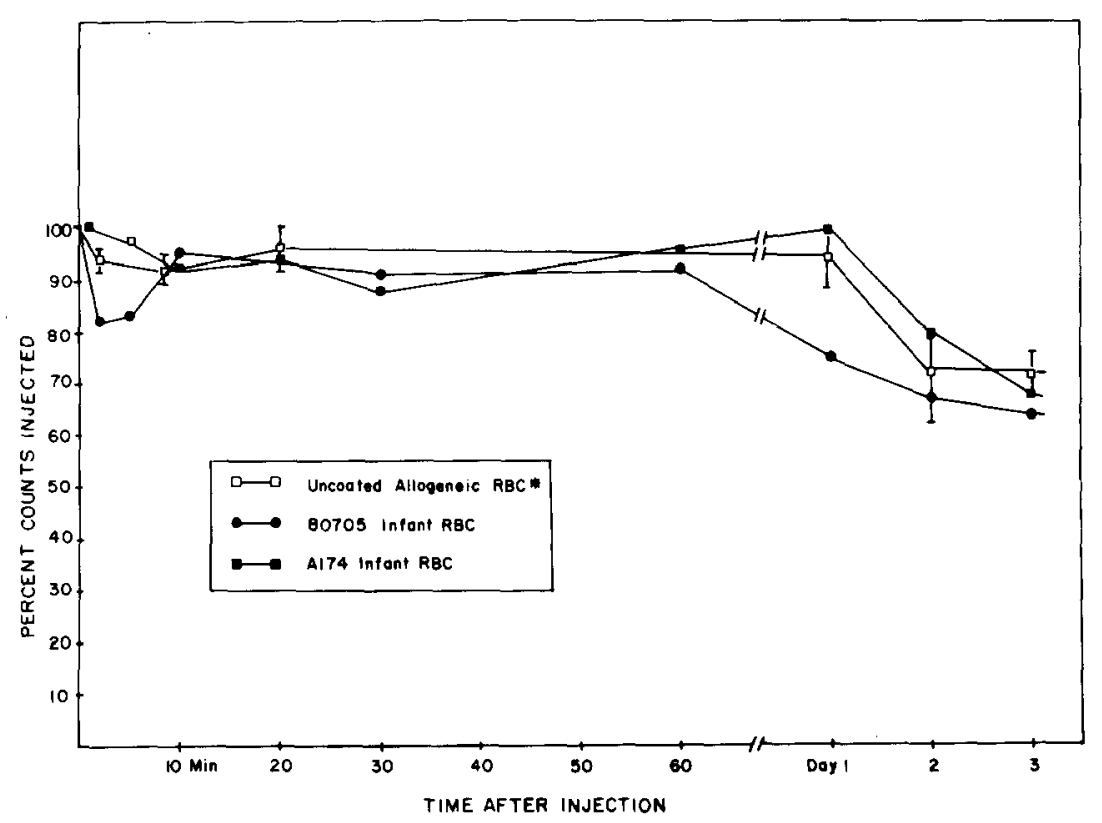

Fig. 1. Survival of rhesus newborn RBC passively coated in utero with maternal antibodies. *Mean \pm S.D. of duplicate experiments. (Reprinted from Stong et al., 1982 with permission from Elsevier Publishing.)

born monkeys to that of uncoated control RBC (derived from adults). The positive DAT indicated that the cells of each newborn had been coated in utero with maternal antibodies. Each mother's postpartum serum titered greater than 1000 against her infant's cells by the modified microtiter agglutination technique. As shown in Figure 1, these antibody-coated RBC survived as well as the uncoated controls: $90 \%$ were circulating 60 min after injection and $65-70 \%$ were still circulating after 3 days. Significantly, these results demonstrated that coated RBC, that apparently survive normally in fetal and newborn monkeys, also survived normally in adult recipients. Moreover, the same pattern of survival was observed with adult RBC that had been coated in vitro using postpartum serum from one of the mothers. In contrast, when radiolabeled human $R B C$ are coated in vitro with anti-D alloantibodies and injected into non-immune volunteers, $30-60 \%$ of the cells are cleared within 30 min, and greater than $90 \%$ are cleared within $2 \mathrm{~h}$ (Mollison, 1983). These results indicate that rhesus maternal antibodies are not opsonic - that is, they do not enhance the clearance of antibodycoated cells from the circulation. This finding is consistent with the absence of HDN in this species.
Effect of rabbit antibodies on erythrocyte survival

In humans the antibodies that cause HDN mediate destruction of fetal RBC by promoting complement-mediated intravascular lysis or by promoting $\mathrm{RBC}$ sequestration in the reticuloendothelial system (RES) (Logue \& Rosse, 1976). To exclude the unlikely possibility of a generalized defect of these systems in rhesus, we tested the ability of rhesus recipients to eliminate cells that had been coated in vitro with purified IgM or IgG rabbit antibodies. We selected rabbit antibodies because in vitro experiments had demonstrated that they were potent agglutinins and hemolysins against rhesus RBC.

Cells that were heavily coated with rabbit IgM ( $30 \mu \mathrm{g} / \mathrm{ml}$ packed cells) exhibited a surprising pattern of survival. Initially, more than $60 \%$ of the cells were rapidly cleared from the circulation. However, most of these cells were then gradually returned to the circulation over the next $24 \mathrm{~h}$, where they continued to circulate in an apparently normal fashion. Similar results have been observed in guinea pigs with IgM-coated cells and in humans with certain alloantibody-coated cells (Frank et al., 1977). 


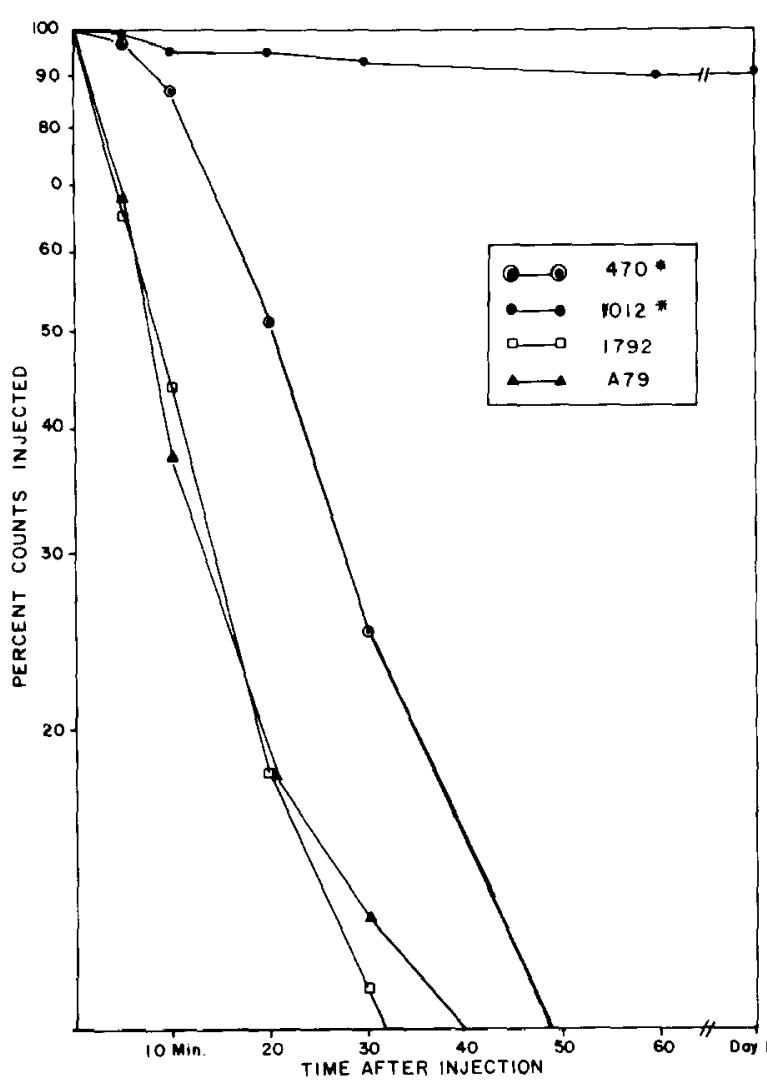

Fig. 2. Survival of incompatible rhesus RBC in hyperimmune recipients. *Representative of duplicate experiments (Reprinted from Stong et al., 1982 with permission from Elsevier Publishing.)

In contrast to the results with rabbit IgM, rabbit IgG readily mediated the clearance of $\mathrm{RBC}$ by rhesus recipients. Significantly, both intravascular hemolysis and extravascular RES sequestration functioned to clear the IgG-coated cells, thereby demonstrating that there is no generalized defect of these rhesus systems. Interestingly, the mechanism of clearance depended upon the concentration of IgG used to coat the cells. When cells were lightly coated with rabbit IgG $(2.4 \mu \mathrm{g} / \mathrm{ml}$ packed $\mathrm{RBC})$, $10 \%$ of the cells were cleared by intravascular hemolysis, and $90 \%$ by extravascular sequestration. When cells were saturated with $\operatorname{IgG}(10 \mu \mathrm{g} / \mathrm{ml}$ packed cells), approximately $55 \%$ of the cells were lysed intravascularly while the remainder were trapped extravascularly by the RES. Taken together, these experiments demonstrate two important points: (1) rhesus monkeys possess two functional mechanisms for eliminating incompatible RBC; and (2) both antibody concentration and immunoglobulin class, IgG versus IgM, are important parameters affecting the fate of antibody-coated RBC.

Effect of rhesus hyperimmune antibodies on erythrocyte survival

We have demonstrated that the maternal antibodies engendered by transplacental immunization do not mediate clearance of rhesus cells, whether coating occurs in vitro or in utero. It seemed reasonable to ask if rhesus antibodies induced by overt hyperimmunization could mediate RBC clearance. Figure 2 presents the results of experiments in which radiolabeled incompatible $\mathrm{RBC}$ derived from different donors were injected into four hyperimmune recipients. Three of the monkeys rapidly eliminated the incompatible cells; less than $2 \%$ of the injected counts were detected in the plasma, indicating that the injected $R B C$ had been cleared by sequestration in the RES. Surprisingly, a fourth monkey, rhesus 1012, did not eliminate the incompatible cells. In separate experiments, incompatible cells were coated in vitro with the hyperimmune antibodies and injected into third party, nonimmune recipients; essentially the same results were observed (results not shown). It is evident that certain rhesus alloantibodies can mediate erythrocyte elimination. However, the antibodies produced by rhesus 1012 resembled maternal antibodies in that they coated incompatible RBC but did not mediate their destruction.

\section{Parameters affecting erythrocyte clearance}

Concentration. It seemed possible that the concentration of rhesus alloantibodies, like that of rabbit IgG antibodies, might play an important role in the clearance of rhesus cells. To test this hypothesis, $\mathrm{RBC}$ were coated in vitro with decreasing concentrations of hyperimmune serum 470 , an antiserum with proven ability to mediate rapid elimination of incompatible cells. A constant volume of serially diluted antiserum was incubated with a $10 \%$ erythrocyte suspension. The survival curves of the coated cells, shown in Figure 3, demonstrate that the amount of antibody influences the rate of clearance and also the total proportion of incompatible RBC cleared. For example, cells that had 
been lightly coated with antibodies (dilution = $1 / 8$ ) survived as well as uncoated cells. In contrast, $90 \%$ of cells coated with only 4 times as many antibodies (dilution $=1 / 2$ ) were cleared within $10 \mathrm{~min}$. In all cases, the cells exhibited a positive DAT, although the strength of the reaction decreased as the antiserum dilution increased. These results strongly suggest that a threshold level of antibody coating is necessary for immune elimination.

Results of these experiments suggested that an inability of maternal antibodies and antiserum 1012 to coat cells adequately might explain their inability to mediate erythrocyte clearance. Measurements of the relative extent of coating by various rhesus alloantibodies supported this hypothesis. Using ${ }^{125}$ I-protein $\mathrm{A}$, we found that RBC saturated with each of the opsonic hyperimmune sera bound 3- to 25 -fold more antibody than the non-opsonic sera (Stong et al., 1984).

Titer and avidity. A comparison of the hyperimmune and several postpartum sera, shown in Table 1 , indicates that antibody titer could not in itself account for the different levels of antibody coating observed for these sera. The titers of all the sera were greater than 1000 by the modified microtiter agglutination method. Neither the classical saline agglutination nor the indirect antiglobulin techniques revealed a significant difference in titer between the two groups. However, it may be significant that more than $50 \%$ of the maternal sera that we have tested by this sensitive method exhibited titers less than 16 (Stong et al., 1984). Experiments with ${ }^{125} \mathrm{I}$-protein $\mathrm{A}$ indicated that once bound to the cells, the antibodies in the hyperimmune sera were more likely to stay attached to the cells than were the antibodies in maternal postpartum sera. In other words, the hyperimmune sera

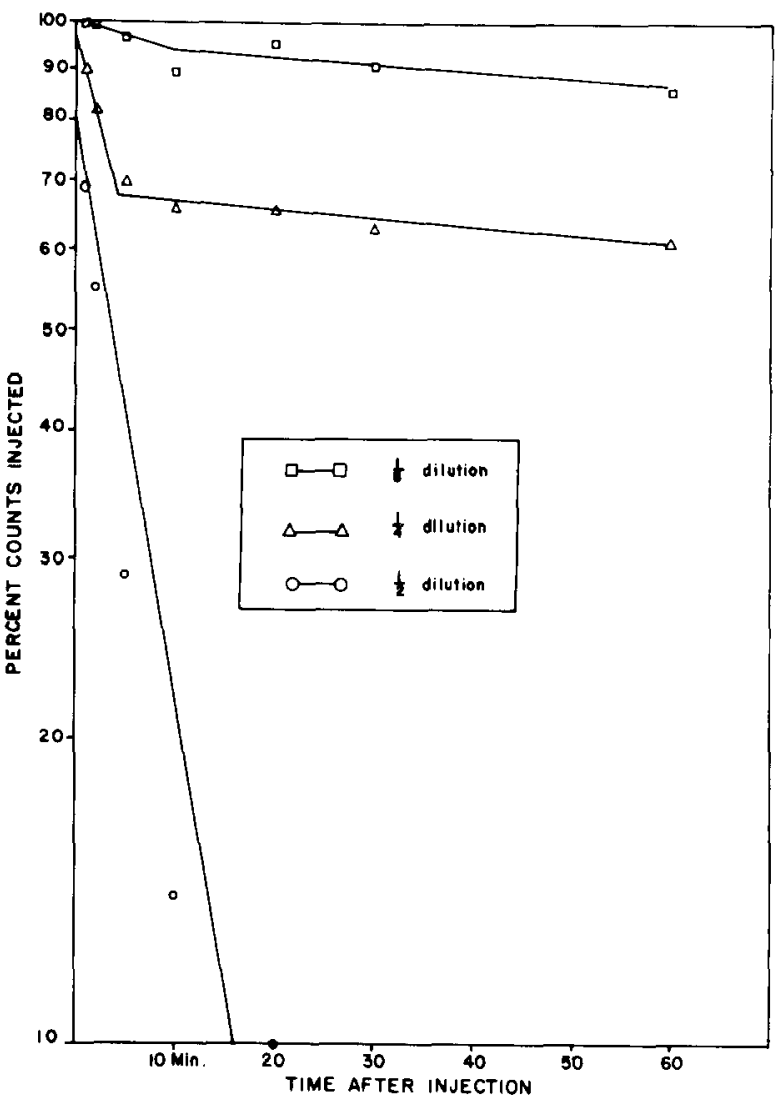

Fig. 3. The effect of antibody concentration on the survival of $\mathrm{RBC}$. Lines for $0-5 \mathrm{~min}$ and for $10-60 \mathrm{~min}$ fit by least squares analysis. (Reprinted from Stong et al., 1982 with permission from Elsevier Publishing.)

contained high avidity antibodies whereas maternal sera contained low avidity antibodies. It seems likely that low titer and low avidity contribute to the inability of maternal antibodies to mediate clearance of the newborn's coated RBC.

Table 1. Titer a and specificity of rhesus alloimmune sera ${ }^{\mathrm{b}}$.

\begin{tabular}{|c|c|c|c|c|c|c|}
\hline & \multicolumn{3}{|c|}{ Opsonic sera } & \multicolumn{3}{|c|}{ Non-opsonic sera } \\
\hline & 470 I.S. & A79 I.S. & 1792 I.S. & 1012 I.S. & 542 M.S. & Z35 M.S. \\
\hline Microtiter agglutination & 8192 & 65536 & 8192 & 32768 & 1024 & 1024 \\
\hline Saline agglutination & 4 & 8 & 4 & 0 & 128 & 4 \\
\hline Indirect agglutination & 512 & 128 & 32 & 128 & $>128$ & 8 \\
\hline Blood group specificities ${ }^{\mathfrak{c}}$ & H1, II & $\mathrm{K}, 38$ & $\mathrm{M1}, 34,38$ & $\mathbf{J}$ & G4 & K \\
\hline
\end{tabular}

a Highest dilution giving $50 \%$ or greater agglutination.

b I.S. = immune serum; M.S. = maternal serum.

c See Stone et al. (1981) for a description of various blood groups: numbered specificities are not published. 
Specificity. One readily apparent difference between the opsonic and the non-opsonic sera (see Table 1) was the number of antibody specificities they contained. All the opsonic sera were multispecific whereas all the non-opsonic sera were monospecific. In general, the number of antibodies bound to several blood group specificities is likely to be greater than the number bound to a single specificity.

Immunoglobulin class and subclass. Phagocytic cells in the RES of humans and other species possess receptors specific for the Fc portion of IgG immunoglobulins; these receptors mediate the removal of antibody-coated RBC from the circulation (Logue \& Rosse, 1976). Such receptors must also function in immune clearance in rhesus monkeys because rhesus RBC that were effectively opsonized were cleared via RES sequestration. It is possible that differences in the immunoglobulin class or subclass of rhesus antibodies could affect their ability to interact with these receptors. $\mathrm{Hu}$ mans, for example, possess $4 \mathrm{IgG}$ subclasses, but only IgG1 and IgG3 interact with human Fc receptors and so are capable of effectively opsonizing RBC.

We separated rhesus alloantisera into purified $\operatorname{IgG}$ and IgM fractions using standard chromatographic techniques and then tested each fraction for its ability to agglutinate RBC. In this way we determined that rhesus alloantibodies in both hyperimmune and postpartum sera belonged primarily to the IgG class (Stong, 1981). It was not possible to detect subclass differences because IgG subclasses had not yet been defined in rhesus monkeys. Therefore, we used typing reagents specific for human IgG subclasses and the direct antiglobulin technique in an attempt to detect differences between the opsonic and non-opsonic hyperimmune sera. We found that the three opsonic sera exhibited medium to strong reactions with three of the four reagents; reactivity with anti-IgG3 was weak and variable. In contrast, the non-opsonic serum (1012) reacted weakly with only two reagents, those detecting IgGl and IgG2. The results suggest that IgG subclass differences exist among the hyperimmune sera and that these differences may relate to their opsonic capability.

Recently, Martin (1982) identified three IgG subclasses in rhesus. The subclasses were separated by ion exchange chromatography and protein $\mathrm{A}$ affini- ty chromatography and then identified antigenicly using rabbit antisera produced against the various fractions. It is now possible using Martin's techniques to examine rigorously in rhesus monkeys the importance of IgG subclass differences in immune elimination of RBC and in HDN. Also, it is possible to address the question of whether the rhesus placenta selectively transfers IgG subclasses which do not bind to $F C$ receptors.

\section{In vitro erythrophagocytosis experiments}

Differences in IgG subclass composition would be important only if rhesus RES receptors did not bind all subclasses equally well. To test this hypothesis we developed an in vitro erythrophagocytosis assay that employed rhesus monkey peripheral blood monocytes (Stong \& Stone, 1984). Monocytes are immature macrophages and are considered to be part of the RES. In brief, our technique consisted of isolating monocytes from venous blood by ficoll-hypaque density centrifugation followed by adherence to glass coverslips. The monocytes were incubated with uncoated or coated RBC for one hour, washed to remove nonadherent $\mathrm{RBC}$, fixed and stained. Monocytes that had ingested or bound at least one $\mathrm{RBC}$ were scored as positive. The results were expressed as the percent positive of total monocytes examined.

We found that rhesus monocytes did not bind or phagocytize uncoated $\mathrm{RBC}$, but did bind and phagocytize RBC that had been coated with partic-

Table 2. Specificity of rhesus Fc receptors for human IgG subclasses.

\begin{tabular}{llll}
\hline $\begin{array}{l}\text { Human } \\
\text { subclass } \\
\text { inhibitor }\end{array}$ & $\begin{array}{l}\text { Conc. } \\
(\mathrm{mg} / \mathrm{ml})\end{array}$ & \multicolumn{2}{l}{ Percent Inhibition $^{\mathrm{a}}$} \\
\cline { 2 - 4 } IgG1 & 0.01 & $98 \pm 4$ & $(89-100)$ \\
& 0.10 & 100 & $(100)$ \\
IgG2 & 0.01 & $31 \pm 25$ & $(0-48)$ \\
& 0.10 & $52 \pm 17$ & $(35-77)$ \\
IgG3 & 0.01 & $93 \pm 7$ & $(81-100)$ \\
& 0.10 & $98 \pm 3$ & $(93-100)$ \\
IgG4 & 0.01 & $47 \pm 15$ & $(9-75)$ \\
& 0.10 & $85 \pm 15$ & $(56-96)$ \\
\hline
\end{tabular}

a Inhibition of erythrophagocytosis determined for 6 monocyte donors. (Modified from Stong \& Stone, 1984). 
Table 3. Comparison of in vivo clearance and in vitro phagocytosis of antibody-coated rhesus erythrocytes.

\begin{tabular}{lll}
\hline $\begin{array}{l}\text { Coating } \\
\text { antibody }\end{array}$ & $\begin{array}{l}\text { Clearance } \\
\text { in vivo }\end{array}$ & $\begin{array}{l}\text { Adherence } \\
\text { in vitro }\end{array}$ \\
\hline $\begin{array}{l}\text { None } \\
\text { Rabbit IgG }\end{array}$ & no $(1.5)^{\mathrm{b}}$ & no $(0.7)^{\mathrm{c}}$ \\
Rabbit IgM & yes $(90)$ & yes $(39)$ \\
Maternal antibodies & no $(35)$ & no $(0.8)$ \\
Rhesus 1012 I.S. & no $(6)$ & no $(0)$ \\
Rhesus 470 I.S. & no $(0.7)$ & no $(2.2)$ \\
Rhesus A79 I.S. & yes $(85)$ & yes $(28)$ \\
Rhesus 1792 I.S. & yes $(94)$ & yes $(18)$ \\
\hline
\end{tabular}

a I.S. = immune serum.

b Percent of injected RBC cleared from circulation within 60 min after injection. (See Stong et al., 1982).

c Percent monocytes binding or phagocytizing RBC. (See Stong \& Stone, 1984).

ular antibodies. For example, rhesus RBC coated with rabbit IgG were readily phagocytized, whereas, IgM-coated RBC were not. If the Fc portion of the IgG antibody was removed by pepsin digestion, the $F\left(a b\right.$ ') ${ }_{2}$-coated $R B C$ were no longer processed. These results demonstrated that rhesus monocytes, like phagocytic cells of other species, possess receptors specific for the Fc portion of IgG.

Human $\mathrm{Fc}$ receptors will bind $\mathrm{IgG1}$ and $\mathrm{IgG} 3$ subclasses, but not IgG2 and IgG4 subclasses (Van der Meulen et al., 1978). To determine if rhesus Fc receptors were similarly restricted, we used purified human IgG subclasses to inhibit competitively the binding and phagocytosis of antibody-coated RBC by rhesus monocytes. The results of these inhibition studies, summarized in Table 2, suggested that rhesus Fc receptors, like human Fc receptors, exhibit restricted subclass specificity.

Table 3 compares the in vivo fate of various antibody-coated RBC to their fate in the in vitro assay. The strong correlation between in vivo and in vitro results demonstrates that the erythrophagocytosis assay is a valid model of antibody-mediated RES sequestration in rhesus monkeys. It should be noted that RBC coated with any of the three opsonic sera were phagocytized whereas newborn's $\mathrm{RBC}$ coated with maternal antibodies and $\mathrm{RBC}$ coated with antiserum 1012 were not.

A number of experiments with our in vitro model confirmed our previous in vivo findings of what parameters are important for RBC clearance (Stong \& Stone, 1984). As an example, dilution ex- periments indicated that a threshold level of antibody coating is necessary for effective interaction between antibody-coated RBC and phagocytic cells. This model provided additional evidence that antibody specificity, and in turn the number of corresponding antigenic sites on the erythrocyte, affects the level of antibody coating. The genotype of the red cell also plays a role because in preliminary experiments, one alloantiserum (anti-H1) mediated phagocytosis of homozygous $(\mathrm{H} 1 / \mathrm{H} 1) \mathrm{RBC}$ but not heterozygous $(\mathrm{H} 1 / \mathrm{H} 2) \mathrm{RBC}$. This finding is relevant to HDN because infants resulting from incompatible matings must be heterozygous for the immunogenic blood group factor.

\section{Conclusions}

Newborn rhesus monkeys do not develop HDN even though their RBC may be coated with maternal antibodies. The results of our studies suggest that the newborn's coated cells survive normally because the maternal antibodies engendered by transplacental immunization are of low titer and low avidity and perhaps are of an IgG subclass that does not bind effectively to the Fc receptors on RES phagocytes. It is tempting to speculate that the absence of an immunodominant blood group antigen that can stimulate potent maternal antibodies may account in large part for the absence of HDN in rhesus monkeys.

\section{Acknowledgments}

The author is the recipient of an Andrew G. Cowles Research Fellowship from the Southwest Foundation for Biomedical Research. Present address: Dr. Robin Stong Treichel, Hematology/ Oncology Research, Children's Hospital of Orange County, 455 S. Main, Orange, CA 92668.

\section{References}

Briles, W. E., 1948. Induced hemolytic disease in chicks. Genetics 33: $96-97$.

Clarke, C. A., 1978. Haemolytic disease of the newborn foal. Proc. R. Soc. Med. 71: 524-585.

Frank, M. M., Schreiber, A. D., Atkinson, J. P. \& Jaffe, C. J., 1977. Pathophysiology of immune hemolytic anemia. Ann. intern. Med. 87: 210-222. 
Fraser, I. D. \& Tovey, G. H., 1976. Observations of Rh isoimmunization: Past, present and future. Clinics Haematol. 5: $149-163$.

Gengozian, N., 1972. A blood factor in the marmoset, Saquinus fuscicollis - its detection, mode of inheritance, and species specificity. J. med. Primatol 1: 272-286.

Gengozian, N., Lushbaugh, C. C., Humason, G. L. \& Kniseley, R. M., 1966. 'Erythroblastosis foetalis' in the primate, Tamarinus nigricollis. Nature, Lond. 209: 731-732.

Giblett, E. R., 1964. Blood group antibodies causing hemolytic disease of the newborn. Clin. Obstet. Gynec. 7: 1044-1055.

Hildemann, W. H., Clark, E. A. \& Raison, R. L., 1981. Comprehensive Immunogenetics. Elsevier North Holland, New York. pp. 118-121.

Issitt, P. D. \& Issitt, C. H., 1977. Applied Blood Group Serology. 2nd Edition, 2nd Printing. Spectra Biologicals, Oxnard, CA. pp. $144-147$.

Landsteiner, K. \& Wiener, A. S., 1940. An agglutinable factor in human blood recognized by immune sera for rhesus blood. Proc. Soc. exp. Biol. Med. 43: 223.

Levine, P., Burnham, L., Katzin, E. M. \& Vogel, P., 1941. The role of iso-immunization in the pathogenesis of erythroblastosis fetalis. Am. J. Obstet. Gynec. 42: 925-931.

Levine, P., Celano, M., Fenichel, R., Pollack, W. \& Singher, H., 1961. A 'D-like' antigen in rhesus monkey, human $R$ h positive and human $\mathrm{Rh}$ negative red blood cells. J. Immun. 87: $747-752$.

Logue, G. \& Rosse, W., 1976. Immunologic mechanisms in autoimmune hemolytic disease. Semin. Hematol. 13: 277-289.

Martin, L. N., 1982. Chromatographic fractionation of rhesus monkey (Macaca mulatta) IgG subclasses using DEAE cellulose and protein A-Sepharose. J. Immun. Meth. 50: 319-329.

Mollison, P. L., 1983. Blood Transfusion in Clinical Medicine. 7th Edition. Blackwell Scientific, Boston. pp. 343-344.

Roberts, G. F., 1957. Comparative Aspects of Hemolytic Disease of the Newborn. William Heinemann, London.

Rodewald, R., 1980. Immunoglobulin transmission in mammalian young and the involvement of coated vesicles. In: Ockleford, C. J. \& Whyte, A. (Eds.) Coated Vesicles. Cambridge University Press, Cambridge. pp. 69-101.

Socha, W. W. \& Moor-Jankowski, J., 1979. Serological maternofetal incompatibility in nonhuman primates. In: Ruppenthal, G. C. (Ed.) Nursery Care of Nonhuman Primates. Plenum, New York. pp. 35-42.

Socha, W. W. \& Ruffie, J., 1983. Blood Groups of Primates: Theory, Practice, Evolutionary Meaning. Monogr. Primatol. 3: $31-98$.

Socha, W. W. \& Van Foreest, A. W., 1981. Erythroblastosis fetalis in a family of captive orangutans. Am. J. Primatol. 1: 326.

Stone, W. H., 1975. Immunogenetic studies of rhesus. In: Bourne, G. H. (Ed.) The Rhesus Monkey. Vol. II, Management, Reproduction, and Pathology. Academic Press, New York. pp. 304-336.

Stone, W. H., VandeBerg, J. L. \& Curie-Cohen, M., 1981. Where are rhesus monkeys on the immunogenetics tree? In: Hildemann, W. (Ed.), Frontiers in Immunogenetics, Elsevier North Holland. pp. 45-60.

Stong, R. C., 1981. Blood group incompatibility and the mechanisms of immune elimination in the rhesus monkey (Macaca mulatta). Ph.D. Dissertation, University of Wiscon$\sin$.

Stong, R. C., Houser, W. D. \& Stone, W. H., 1982. The absence of hemolytic disease in the newborn rhesus monkey (Macaca mulatta). Vet. Immun. Immunopathol, 3: 611-627.

Stong, R. C. \& Stone, W. H., 1984. Mononuclear phagocyte receptors in rhesus monkeys (Macaca mulatta) and their role in hemolytic disease of the newborn. Vet. Immun. Immunopathol. 7: 101-117.

Stong, R. C., Nelson, K. H. \& Stone, W. H., 1984. Transplacental alloimmunization to fetal erythrocytes in rhesus monkeys (Macaca mulatta). Am. J. reprod. Immun. 6: 128-132.

Stormont, C., 1975. Neonatal isoerythrolysis in domestic animals: A comparative review. Adv. vet. Sci. comp. Med. 19 $23-45$.

Sullivan, P. T., Blystad, C. \& Stone, W. H., 1977. Immunogenetic studies of rhesus monkeys. VII. A simple hemagglutination technique for blood typing. J. Immun. Meth. 14: 31-36.

Sullivan, P. T., Duggleby, C. R., Blystad, C. \& Stone, W. H., 1976. Immunogenetic studies of rhesus monkeys. V. Transplacental immunization due to blood group incompatibilities. Immunogenetics 3: 473-479.

Van der Meulen, F. W., Van der Hart, M., Fleur, A. \& Von dem Borne, A. E. G. Kr., 1978. The role of adherence to human mononuclear phagocytes in the destruction of red cells sensitized with non-complement binding IgG antibodies. Br. J. Haemat. 38: $541-550$.

Verbickij, M. S., 1972. The use of hamadryas baboons for the study of the immunological aspects of human reproduction. Acta endocr., Copenh. (Suppl.) 166: 492-505.

Verbitsky, M. S., Volkova, L. S., Kuksova, M. I., Lapin, B. A., Andreeva, A. V. \& Gvazava, I. S., 1969. A study of haemolytic disease of the foetus and the newborn occurring in Hamadryas baboons under natural conditions. Z. Versuchstierkd. B 11,S: 136-145.

Voak, D., 1969. The pathogenesis of ABO haemolytic disease of the newborn. Vox Sang. 17: 481-513.

Wiener, A. S., Gavin, J. A. \& Gordon, E. B., 1953. Blood group factors in anthropoid apes and monkeys. II. Further studies on the Rh-hr factors. Am. J. phys. Anthrop. 11: 39-45.

Wiener, A. S., Socha, W. W. \& Gordon, E. B., 1971. Fractionation of human anti-Rho sera by absorption with red cells of apes. Haematologia 5: 227-240.

Wiener, A. S., Socha, W. W. \& Moor-Jankowski, J., 1977. Erythroblastosis models. I1. Maternal-fetal incompatibility in chimpanzee. Folia Primatol. 27: 68-74.

Wiener, A. S., Socha, W. W., Niemann, W. \& Moor-Jankowski, J., 1975. Erythroblastosis models. A review and new experimental data in monkeys. J. med. Primatol. 4: 179-187. 